\title{
How Useful the Combined Impedance-pH Monitoring Is for Evaluation of Refractory Reflux Symptoms?: Author's Reply
}

TO THE EDITOR: We would like to appreciate the interest shown on our study by Dr. Cho. ${ }^{1}$ Our response to her queries is as follows:

(1) We do agree that doubling the proton pump inhibitor (PPI) dose could be the most appropriate therapeutic strategy for gastroesophageal reflux disease (GERD) patients who are refractory to single dose of PPI before performing a $\mathrm{pH}$-impedance measurement. However, previous studies challenged this approach and showed the majority of patients with persistent GERD symptoms on single PPI dose to experience symptoms continuously on even higher doses of PPIs. ${ }^{2,3}$ Our results showed that even though patients on standard PPI dose continued to report more symptoms during the study, the PPI dose seemed to have no impact on the symptom index analysis. ${ }^{4}$ This finding could imply that increase of the PPI dose should not be the solution in patients with refractory GERD like symptoms and that other causes (ie, functional heartburn and hypersensitive esophagus) could explain the origin of symptoms.

Moreover, in every day clinical practice, escalation of the dose is sometimes problematic. In our study, the majority of our population was referred by others and the low rate of double dose PPI usage reflects the absence of reimbursement for PPIs above the standard dose in Greece.

(2) We also agree that in GERD patients with atypical symptoms, $\mathrm{pH}$-impedance monitoring is better to be performed while they are off medication. As we had mentioned in the article, our patient population was inhomogeneous. Many patients reported both typical and atypical symptoms, whereas others reported only atypical symptoms. It is clear that this practice influenced the sensitivity and clinical impact of $\mathrm{pH}$-impedance monitoring. Choosing the most prevalent symptom could be a better surrogate index. However, it is widely accepted that the majority of patients report more than one symptom. Large population-based studies have shown presence of ENT and pulmonary symptoms among patients with reflux symptoms. ${ }^{5,6}$

As $\mathrm{pH}$-impedance monitoring is a new technique that tries to find its position in the everyday clinical practice, further studies are needed to evaluate its clinical impact and usefulness.

Georgios Karamanolis

Hepatogastroenterology Unit 2nd Department of Internal Medicine - Propaedeutic Attikon University General Hospital Athens Medical School, Athens, Greece

1. Cho YK. How useful the combined impedance-pH monitoring is for evaluation of refractory reflux symptoms? J Neurogastroenterol Motil 2011;17:327-328.

2. Klinkenberg-Knol EC, Festen HP, Jansen JB, et al. Long-term treatment with omeprazole for refractory reflux esophagitis: efficacy and safety. Ann Intern Med 1994;121:161-167.

3. Leite LP, Johnston BT, Just RJ, Castell DO. Persistent acid secretion during omeprazole therapy: a study of gastric acid profiles in patients demonstrating failure of omeprazole therapy. Am J Gastroenterol 1996;9:1527-1531.

4. Karamanolis G, Kotsalidis G, Triantafyllou K, et al. Yield of combined impedance- $\mathrm{pH}$ monitoring for refractory reflux symptoms in clinical practice. J Neurogastroenterol Motil 2011;17:158-163.

5. Locke RG 3rd, Talley NJ, Fett SL, Zinsmeister AR, Melton LJ 3rd. Prevalence and clinical spectrum of gastro-esophageal reflux: a population-based study in Olmsted county, Minnesota. Gastroenterology 1997;112:1448-1456.

6. El-Serag HB, Sonnenberg A. Comorbid occurrence of laryngeal or pulmonary disease with esophagitis in United States military veterans. Gastroenterology 1997;113:755-760.

\section{Conflicts of interest: None.}

